\title{
Numerical Approach of the Ciliary Muscle Contraction. A Preliminary Step to Reproduce Human Accommodation
}

\author{
I.Cabeza Gil ${ }^{1}$, J. Grasa ${ }^{1}$, A.Glasser ${ }^{2}$, B. Calvo ${ }^{1}$ \\ ${ }^{1}$ Afiliación: Applied Mechanics and Bioengineering (AMB) \\ Instituto de Investigación en Ingeniería de Aragón (I3A) \\ Universidad de Zaragoza, Mariano Esquillor s/n, 50018, Zaragoza, Spain. \\ Tel.+34-658403556, e-mail: iulen@unizar.es \\ ${ }^{2}$ Asesor
}

\section{Summary}

The ciliary muscle contraction was simulated through an electro-mechanical continuum model adapted to smooth muscle. The numerical contraction was compared and calibrated accurately according to experimental in vivo data. Moreover, two in vivo subjective measurement techniques to obtain the profile of the ciliary muscle thickness were compared.

\section{Introduction}

Accommodation is the ability of the eye to change its focus from distant to near objects (and vice versa). This process is achieved by changing the shape of the lens, which occurs after the contraction of the ciliary muscle, which allows the release of zonular tension around the lens equator, according to the widely accepted accommodation theory of Helmholtz.

In the literature, ciliary muscle contraction has been measured in vivo in the living eye via optical coherence tomography (OCT), which provides profiles of the ciliary muscle thickness (CMT) along the overall length of the muscle ${ }^{1,2}$. However, measuring in vivo ciliary muscle contraction is still a challenge for various reasons, i.e. no reference position can be set for the subject and the difficulty to extract the small changes in microns that occur. So far two techniques based on OCT imaging have emerged to measure the CMT profiles.

In this study, we performed the numerical contraction of the ciliary muscle by means of a continuum electro-mechanical skeletal continuum model adapted to smooth muscle ${ }^{3}$. The purpose is two-fold: on the one hand, to simulate faithfully the ciliary muscle contraction and on the other hand, to compare two previously published experimental measurement techniques of the CMT profiles ${ }^{2,4}$.

\section{Materials \& Methods}

The geometry of the average emmetrope ciliary muscle reported by Wagner et al. ${ }^{1}$ was used in our finite element (FE) model. The ciliary muscle was arranged into circular, radial and longitudinal muscle fibres, with fibre volumes of $12 \%, 33 \%$ and $55 \%$, respectively, according to literature ${ }^{5}$, see Fig. 1.

In terms of the passive and active finite strain response of the muscle, the ciliary muscle was simulated within the framework of continuum mechanics using a very common methodology based on postulating the existence of a strain energy function ${ }^{6}$ :

$$
\Psi=\Psi(C, C e, \lambda a, N, M)
$$

This function depends on the state variables $\mathbf{F}$ (deformation gradient), $\lambda \mathrm{a}$ (contraction or stretch of the muscle fibres) and of the structural tensors $\mathbf{M}=\mathrm{m}_{0} \otimes \mathrm{m}_{0}$ and $\mathbf{N}=\mathrm{n}_{0} \otimes \mathrm{n}_{0}$ which define the anisotropy of the muscle due to the preferential direction of muscular and collagen fibres, respectively. The function also depends on $\mathrm{Ce}$ which represents the elastic deformation of the cross-bridge.

Moreover, two measurement techniques to obtain the CMT profile along the overall length of the muscle were used and compared. One measured the thickness from perpendicular rays of the sclera and another measured the minimum thickness from the outer boundary of the ciliary muscle to the sclera $^{1,2,3}$, see Fig. 2.

\section{Results}

The morphology of the numerical ciliary muscle contraction for an accommodated stimulus of 4 dioptres (D) is compared accurately with experimental data ${ }^{1}$, see Fig. 3. Moreover, a methodology to calibrate the main ciliary muscle properties which control the temporal performance of the muscle contraction is presented. 
Regarding the comparison of the numerical results using both OCT ciliary muscle profile measurement methods, slight differences were obtained for a small amount of accommodation, which are related to small reduction in the ciliary muscle ring diameter. Nevertheless, significant differences in the numerical results were obtained using both methods for a larger amount of accommodation.

\section{Conclusions}

- The morphology of the ciliary muscle contraction was accurately reproduced by a electro-mechanical continuum model adapted to smooth muscles.

- Ciliary muscle properties were calibrated according to experimental in vivo data.

- Numerical ciliary muscle contraction was compared using both qualitative OCT measurement techniques.

\section{REFERENCIAS}

[1]. Wagner, S., Zrenner, E., Strasser, T. Emmetropes and myopes differ little in their accommodation dynamics

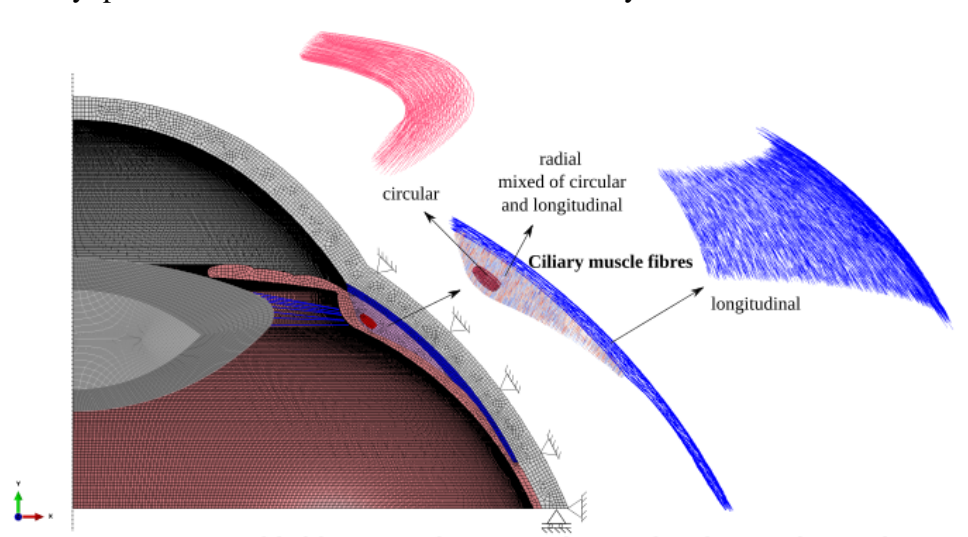

Fig.1. 3D FE model of the accommodative system: lens, zonules, ciliary muscle, iris, sclera and cornea. The arrangement and orientation of theciliary muscle fibres, longitudinal (blue), radial (red-blue gradient) and circumferential (red), is shown at the right. but strongly in their ciliary muscle morphology. Vision Research. 2019, 163, 42-51.

[2]. Ruggeri, M., de Freitas, C., Williams, S., Hernandez, V. M., Cabot, F., Yesilirmak, N., Alawa, K., Chang, Y.-C., Yoo, S. H., Gregori, G., Parel, J.-M., Manns, F. Quantification of the ciliary muscle and crystalline lens interaction during accommodation with synchronous OCT imaging. Biomedical Optics Express. 2016, 7 (4), 1351.

[3]. Hernández-Gascón, B., Grasa, J., Calvo, B., Rodríguez, J.F. A 3d electro-mechanical continuum model for simulating skeletal musclecontraction. Journal of Theoretical Biology. 2013, 335, 108-18.

[4]. Straßer, T., Wagner, S., Zrenner, E. Review of the application of the open-source software CilOCT for semi-automatic segmentation and analysis of the ciliary muscle in OCT images. PLOS ONE. 2020, 15 (6), e0234330.

[5]. Pardue, M. T., Sivak, J. G. Age-related changes in human ciliary muscle. Optometry and Vision Science. 2000, 77 (4), 204-210.

[6]. Grasa, J., Sierra, M., Lauzeral, N., Muñoz, M., Miana-Mena, F., Calvo, B. Active behavior of abdominal wall muscles: Experimental results and numerical model formulation. Journal of the Mechanical Behavior of Biomedical Materials. 2016, 61, 444-454.

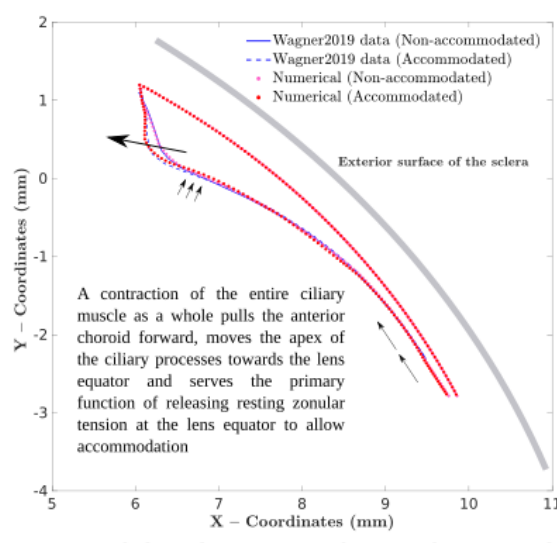

Fig.3. Morphological Comparative between the numerical and experimental contraction of the ciliary muscle.
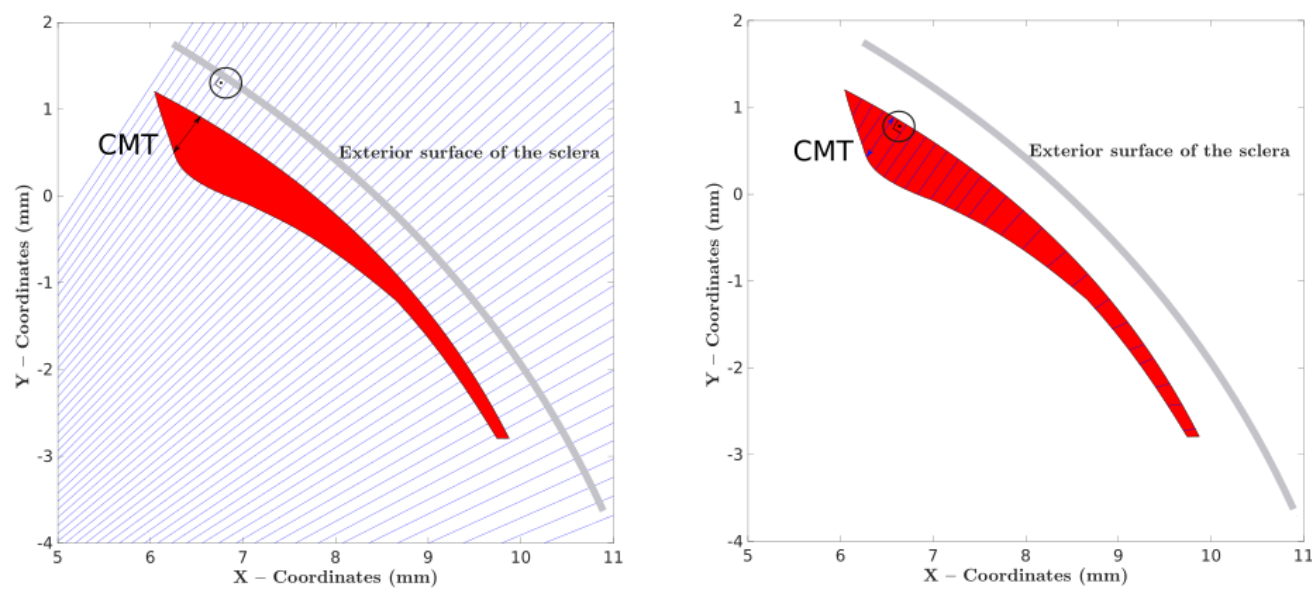

Fig.2. Two measurement techniques used to evaluate CMT profiles. On the left, the CMT is measured from perpendicular rays from the sclera. On the right, the CMT is the minimum distance from the outer boundary of the ciliary muscle to the sclera.

Revista "Jornada de Jóvenes Investigadores del I3A", vol. 8 (Actas de la IX Jornada de Jóvenes Investigadores del I3A -11 de diciembre de 2020). ISSN 2341-4790. 